\title{
SIMULATION STUDY OF FISH SWIMMING MODES FOR AQUATIC ROBOT SYSTEM
}

\author{
EunJung Kim \& Youngil Youm*
}

* Dept.of M.E.,POSTECH, Pohang, Korea

\begin{abstract}
In this paper, we show the simulation result to find the suitable fish swimming modes(specially BCF swimming) for fishlike underwater robot system. To find the suitable swimming modes, we assume that they have the same length, volume, and weight, but the different numbers of actuator(joint). And we use the minimum number of the joint for each swimming mode. We derive the dynamic equation for each system using Kane's method and these results are compared by DADS. We present the optimal solution of swimming mode for some aquatic locomotion, especially faster(high propulsive efficiency) and more maneuverable(quick turning motion). Copyright (C2005 IFAC
\end{abstract}

Keywords: Carangiform locomotion, Underwater vehicle, Kane's Dynamics

\section{INTRODUCTION}

Many research group used the propellers to propel the underwater vehicles. But some researchers used the tail or the overall shape of the body as fish because of high propulsive efficiency, great maneuverability, and good stealth recently [Barrett, 2002], [Anderson, 2002]. In this paper, we study many fish swimming modes (especially BCF swimming mode) [Sfakiotakis, 1999] to design aquatic robot system with high propulsion speed and quick turning motion. The motivation is to provide a relevant and useful data for engineers involved in underwater vehicle design and control and for those with an interest in the fast-growing area of biomimetic swimming robots. The classification of swimming movements presented here adopts the (expanded) nomenclature originally put forth by Breder in [Breder,1926]. Most fish generate thrust by bending their bodies into a backward-moving propulsive wave that extends to its caudal fin, a type of swimming classified under body and/or caudal fin(BCF) locomotion. Other fish have developed alternative swimming mechanisms that involve the use of their median and pectoral fins, termed median and/or paired
fin(MPF) locomotion. In this paper, we concentrate on the BCF locomotion because of their propulsion ability. The advantages of improved propulsive efficiency, stealth, turning radii, and acceleration could prove to be significant if applied to the design of fishlike underwater robot system. Because we suggest the good swimming mode for high propulsion speed or quick turning motion, we derive the dynamic equation for each swimming mode using Kane's method [Kane, 1983]. For simplicity, we assume that the minimum number of joints for embodying each fish swimming mode is used. And we assume that each mode has the same length, weight, and volume. Their ability for propulsive efficiency(high speed) and good turning radii(quick turning) can be compared by supplying the same input power. In Section II, we will explain the dynamic equation for each swimming mode using Kane's method. In Section III, there will be dynamic simulation result compared by DADS. In this section we will check the feature for each fishlike swimming mode and present good design mode for suitable locomotion, especially high propulsive efficiency and quick turning mo- 


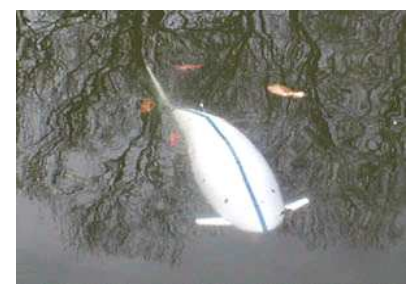

Fig. 1. Fish robot PoTuna

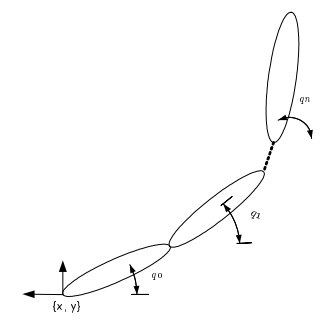

Fig. 2. The 2 dimensional Coordinate frame of fish robot using Kane's method

tion. In Section IV, there will be summary about this paper.

\section{DYNAMIC EQUATION USING KANE'S METHOD}

In this section, we will develop a dynamic model for each swimming mode using Kane's dynamic equation. We assume that fish robot has $\mathrm{n}$ links. We will select the $N=6+n$ generalized velocities of the system as:

$$
\dot{\mathbf{q}}=\left[\begin{array}{lllllllll}
v_{x} & v_{y} & v_{z} & \omega_{x} & \omega_{y} & \omega_{z} & \dot{\theta}_{1} & \cdots & \dot{\theta}_{n}
\end{array}\right]^{T}
$$

where $\left(v_{x}, v_{y}, v_{z}\right)$ is the linear velocity of the fish robot with respect to the inertial frame expressed in the $B$ th coordinate frame, $\left(\omega_{x}, \omega_{y}, \omega_{z}\right)$ is the angular velocity of the fish robot with respect to the inertial frame expressed in the $B$ th coordinate frame, and $\left(\dot{\theta}_{1}, \cdots, \dot{\theta}_{n}\right)$ which are the joint velocities of each link. Figure 2 show the 2 dimensional coordinate frame of fish robot using Kane's method [Tarn, 1996], respectively.

\subsection{Kinematic Analysis}

The kinematic task is more tractable and systematic if we exploit the use of Denavit-Hartenberg(D$\mathrm{H})$ coordinate frames. Figure 2 illustrates the coordinates that we have selected for our system. The position vector of the C.M. of link 1 with respect to the C.M. of the fish body expressed in the $B$ coordinate frame is given by:

$$
\mathbf{p}_{1}^{B}=\left[\begin{array}{c}
c_{x_{B}} \\
c_{y_{B}} \\
c_{z_{B}} \\
1
\end{array}\right] \tilde{+} \mathbf{A}_{B}^{1} \mathbf{c}_{1}^{1}=\mathbf{c}_{B}^{B} \tilde{+} \mathbf{A}_{B}^{1} \mathbf{c}_{1}^{1}
$$

where $\tilde{+}$ denote the addition of the physical coordinates of the two homogeneous vectors on the right hand side, $\mathbf{A}_{B}^{1}$ is the homogeneous transformation from coordinate frame 1 to coordinate frame $B$, and $\mathbf{c}_{1}^{1}$ is the position vector to the C.M. of link 1 expressed in frame 1 . The position vector of the C.M. of the link 2 with respect to the C.M. the body is given similarly by:

$$
\mathbf{p}_{2}^{B}=\mathbf{c}_{B}^{B} \tilde{+} \mathbf{A}_{B}^{1} \mathbf{A}_{1}^{2} \mathbf{c}_{2}^{2}
$$

and for an arbitrary link $j, j=1, \cdots, n$ by the following:

$$
\mathbf{p}_{j}^{B}=\mathbf{c}_{B}^{B} \tilde{+} \prod_{k=1}^{j} \mathbf{A}_{k-1}^{k} \mathbf{c}_{j}^{j}
$$

The angular velocity of the fish body with respect to an inertial frame $E$, expressed in the $B$ th coordinate system is given by:

$$
{ }^{E} \boldsymbol{\omega}^{B}=\boldsymbol{\omega}_{x} \hat{x}_{B}+\boldsymbol{\omega}_{y} \hat{y}_{B}+\boldsymbol{\omega}_{z} \hat{z}_{B}
$$

where the hat denotes a unit vector and the notation ${ }^{E} \boldsymbol{\omega}^{B}$ denotes the angular velocity of frame $B$ with respect to frame $E$ expressed in the $B$ th coordinate frame. The angular velocity of peduncle and tail with respect to the inertial frame expressed in the $B$ th coordinate frame is given by:

$$
\begin{aligned}
{ }^{E} \boldsymbol{\omega}^{1} & ={ }^{E} \boldsymbol{\omega}^{B}+{ }^{B} \boldsymbol{\omega}^{1}={ }^{E} \boldsymbol{\omega}^{B}+\dot{\theta}_{1} \hat{z}_{B} \\
{ }^{E} \boldsymbol{\omega}^{2} & ={ }^{E} \boldsymbol{\omega}^{B}+{ }^{B} \boldsymbol{\omega}^{1}+\overline{\mathbf{A}}_{B}^{1}{ }^{1} \boldsymbol{\omega}^{2} \\
& ={ }^{E} \boldsymbol{\omega}^{B}+{ }^{B} \boldsymbol{\omega}^{1}+\overline{\mathbf{A}}_{B}^{1} \dot{\theta}_{2} \hat{z}_{1} \\
& \vdots \\
{ }^{E} \boldsymbol{\omega}^{j} & ={ }^{E} \boldsymbol{\omega}^{B}+{ }^{B} \boldsymbol{\omega}^{1}+\sum_{l=1}^{j-1} \prod_{m=1}^{l} \overline{\mathbf{A}}_{m-1}^{m}{ }^{l} \boldsymbol{\omega}^{l+1}
\end{aligned}
$$

where $\overline{\mathbf{A}}_{B}^{1}$ denotes the rotation submatrix of $\mathbf{A}_{B}^{1}$, ${ }^{B} \boldsymbol{\omega}^{1}$ is the angular velocity of the link 1 with respect to body and ${ }^{1} \boldsymbol{\omega}^{2}$ is the angular velocity of the link 2 with respect to the link 1 . The linear velocity of the C.M. of the body with respect to the inertial frame, expressed in the $B$ th coordinate system is given by:

$$
\mathbf{v}_{B}^{B}=v_{x} \hat{v}_{B}+v_{y} \hat{y}_{B}+v_{z} \hat{z}_{B}
$$

The linear velocity of the C.M. of an arbitrary link $j$ with respect to the inertial frame, expressed in the $B$ th coordinate frame is given by:

$$
\mathbf{v}_{j}^{B}=\mathbf{v}_{B}^{B}+\frac{d \mathbf{p}_{j}^{B}}{d t}+{ }^{E} \boldsymbol{\omega}^{B} \times \mathbf{p}_{j}^{B}
$$

The angular acceleration about the C.M. of the body with respect to the inertial frame, expressed in the $B$ th coordinate frame is given by:

$$
{ }^{E} \boldsymbol{\alpha}^{B}=\dot{\omega}_{x} \hat{x}_{B}+\dot{\omega}_{y} \hat{y}_{B}+\dot{\omega}_{z} \hat{z}_{B}
$$

The angular acceleration of an arbitrary link $j$ with respect to the inertial frame expressed in the $B$ th coordinate frame is found from the following:

$$
{ }^{E} \boldsymbol{\alpha}^{j}=\frac{d^{E} \boldsymbol{\omega}^{j}}{d t}+{ }^{E} \boldsymbol{\omega}^{B} \times{ }^{E} \boldsymbol{\omega}^{j}
$$


The linear acceleration of the C.M. of the body with respect to the inertial frame, expressed in the $B$ th coordinate frame is given by:

$$
\mathbf{a}_{B}^{B}=\frac{d \mathbf{v}_{B}^{B}}{d t}+{ }^{E} \boldsymbol{\omega}^{B} \times \mathbf{v}_{B}^{B}
$$

The linear acceleration of the C.M. of an arbitrary link $j$ with respect to the inertial frame expressed in the $B$ th coordinate frame is found similarly by the following:

$$
\mathbf{a}_{j}^{B}=\frac{d \mathbf{v}_{j}^{B}}{d t}+{ }^{E} \boldsymbol{\omega}^{B} \times \mathbf{v}_{B}^{j}
$$

where $j$ denotes an $j$ th link.

\subsection{Inertia Forces}

The generalized inertia force of the system requires that we develop expressions for the inertia force and torque of each part in the system. The inertia force of an arbitrary link $j$ is given by the following:

$$
\mathbf{R}_{j}^{*}=-m_{j} \mathbf{a}_{j}^{B}
$$

where $m_{j}$ is the mass of fish body, peduncle or tail. The inertia torques of an arbitrary link $j$ are given by the following:

$$
\mathbf{T}_{j}^{*}=-\mathbf{I}_{j}^{B} \cdot{ }^{E} \boldsymbol{\alpha}^{j}-{ }^{E} \boldsymbol{\omega}^{j} \times \mathbf{I}_{j}^{E} \cdot{ }^{E} \boldsymbol{\omega}^{j}
$$

where $\mathbf{I}_{j}^{B}$ is the central inertia matrix of fish body, peduncle or tail, expressed in the $B$ th coordinate frame. The generalized inertia force for the system is now found to obtain the following:

$$
\begin{gathered}
\mathbf{F}_{r}^{*}=\sum_{i=B}^{n}\left(\frac{\partial^{E} \boldsymbol{\omega}^{i}}{\partial \dot{q}_{r}} \cdot \mathbf{T}_{i}^{*}+\frac{\partial \mathbf{v}_{i}^{B}}{\partial \dot{q}_{r}} \cdot \mathbf{R}_{i}^{*}\right) \\
(r=1, \cdots, N)
\end{gathered}
$$

\subsection{Gravity Forces}

Gravity can be treated as a generalized active force which acts at the center of mass of each part in the system. The force due to gravity acting on the $j$ th link is given by:

$$
\mathbf{R}_{\operatorname{grav}_{j}}=m_{j} \mathbf{g}^{B}
$$

where $\mathbf{g}^{B}=\left[g_{x}, g_{y}, g_{z}\right]^{T}$ is the gravity vector expressed in the $B$ th coordinate frame. The generalized active force due to gravity is given by the following:

$$
\left(\mathbf{F}_{r}\right)_{\text {gravity }}=\sum_{i=B}^{n} m_{i} \frac{\partial \mathbf{v}_{i}^{B}}{\partial \dot{q}_{r}} \cdot \mathbf{g}^{B}
$$

\subsection{Hydrodynamic Forces}

The hydrodynamic forces induced by the motion of a rigid body in an underwater environment are very complex and highly nonlinear. The forces may be developed using incompressible fluid flow using Navior-Stokes equation, and rarely lead to a closed form solution. As is often the case, these forces may be treated as lumped approximations for certain applications within certain underlying assumptions. The net effect of added mass, buoyancy, Froude-Kriloff, and drag are often treated as the superposition of each individual force. The added mass force results from the interaction of fluid in the immediate vicinity of a submerged link which is acceleration on the fluid through a pressure distribution which acts on the link body. The force required to accelerate the surrounding fluid results in an effective inertia which can be modeled with a $6 \times 6$ positive definite added mass inertia matrix, $\mathbf{I}_{A}$. In general the 36 elements of the added mass matrix, $\mathbf{I}_{A}$, for a body in a real fluid would be distinct and may be determined from experimental testing techniques. It has been shown by McMillan et al. [Kane, 1983] and can be derived from Fossen [Fossen, 1994], that the inertia force and torque of a submerged body induced by the added mass phenomena has the following form:

$$
\left[\begin{array}{l}
\mathbf{R}_{A_{i}}^{*} \\
\mathbf{T}_{A_{i}}^{*}
\end{array}\right]=-\mathbf{I}_{A_{i}}^{B}\left[\begin{array}{c}
\dot{\mathbf{v}}_{i}^{B} \\
{ }^{E} \boldsymbol{\omega}^{i}
\end{array}\right]-\left[\begin{array}{cc}
E \tilde{\boldsymbol{\omega}}^{i} & 0 \\
\tilde{\mathbf{v}}_{i}^{B} & E \tilde{\boldsymbol{\omega}}^{i}
\end{array}\right] \mathbf{I}_{A_{i}}^{B}\left[\begin{array}{c}
\mathbf{v}_{i}^{B} \\
E \boldsymbol{\omega}^{B, P, T}
\end{array}\right]
$$

where ${ }^{E} \tilde{\boldsymbol{\omega}}^{i}$ and $\tilde{\mathbf{v}}_{i}^{B}$ are skew symmetric matrices, and $\mathbf{I}_{A_{i}}^{B}$ is the $6 \times 6$ added mass matrix for an arbitrary link $i$ expressed in the $B$ th coordinate frame. Substituting these two equations into results

$$
\begin{aligned}
& {\left[\begin{array}{c}
\mathbf{R}_{A_{i}}^{*} \\
\mathbf{T}_{A_{i}}^{*}
\end{array}\right]=-\mathbf{I}_{A_{i}}^{B}\left[\begin{array}{c}
\mathbf{a}_{i}^{B} \\
{ }^{E} \boldsymbol{\alpha}^{i}
\end{array}\right]+\mathbf{I}_{A_{i}}^{B}\left[\begin{array}{c}
{ }^{E} \boldsymbol{\omega}^{i} \times \mathbf{v}_{i}^{B} \\
{ }^{E} \boldsymbol{\omega}^{i} \times{ }^{E} \boldsymbol{\omega}^{i}
\end{array}\right]} \\
& -\left[\begin{array}{cc}
E \tilde{\boldsymbol{\omega}}^{i} & 0 \\
\tilde{\mathbf{v}}_{i}^{B} & { }^{E} \tilde{\boldsymbol{\omega}}^{i}
\end{array}\right] \mathbf{I}_{A_{i}}^{B}\left[\begin{array}{c}
\mathbf{v}_{i}^{B} \\
{ }^{\boldsymbol{\omega}^{i}}
\end{array}\right]
\end{aligned}
$$

We can account for the relative acceleration and velocity of the fluid by introducing the following relationship:

$$
\begin{aligned}
& \mathbf{v}_{i}^{r}=\mathbf{v}_{i}^{B}-\mathbf{v}_{f}^{B} \\
& \mathbf{a}_{i}^{r}=\mathbf{a}_{i}^{B}-\mathbf{a}_{f}^{B}
\end{aligned}
$$

where, $\mathbf{v}_{f}^{B}$ is the velocity of the fluid expressed in the $B$ th coordinate frame, and $\mathbf{a}_{f}^{B}$ is the acceleration of the fluid expressed in the $B$ th coordinate frame. The final form of the inertia force and torque resulting from added mass is now given by: 


$$
\begin{aligned}
{\left[\begin{array}{c}
\mathbf{R}_{A_{i}}^{*} \\
\mathbf{T}_{A_{i}}^{*}
\end{array}\right]=} & -\mathbf{I}_{A_{i}}^{B}\left[\begin{array}{c}
\mathbf{a}_{i}^{r} \\
{ }^{E}{ }^{i}{ }^{i}
\end{array}\right]+\mathbf{I}_{A_{i}}^{B}\left[\begin{array}{c}
{ }^{E} \boldsymbol{\omega}^{i} \times \mathbf{v}_{i}^{r} \\
{ }^{E} \boldsymbol{\omega}^{i} \times{ }^{E} \boldsymbol{\omega}^{i}
\end{array}\right] \\
& -\left[\begin{array}{cc}
E \tilde{\boldsymbol{\omega}}^{i} & 0 \\
\tilde{\mathbf{v}}_{i}^{r} & E \\
\tilde{\boldsymbol{\omega}}^{i}
\end{array}\right] \mathbf{I}_{A_{i}}^{B}\left[\begin{array}{c}
\mathbf{v}_{i}^{r} \\
{ }^{E} \boldsymbol{\omega}^{i}
\end{array}\right]
\end{aligned}
$$

The generalized inertia force due to the added mass for the entire system is then given by the following:

$$
\left(\mathbf{F}_{r}^{*}\right)_{\mathrm{AM}}=\sum_{i=B}^{n}\left(\frac{\partial^{E} \boldsymbol{\omega}^{i}}{\partial \dot{q}_{r}} \cdot \mathbf{T}_{A_{i}}^{*}+\frac{\partial \mathbf{v}_{i}^{B}}{\partial \dot{q}_{r}} \cdot \mathbf{R}_{A_{i}}^{*}\right)
$$

This is a general formulation for the incorporation of the hydrodynamic force and torque into the dynamic model. No assumptions were necessary on how the coefficients of the added mass matrix are derived. The buoyancy force is proportional to the mass of the fluid displaced by the fish body and acts through the center of buoyancy of the each link. For a homogeneous symmetric shape, the center of buoyancy and center of mass are equivalent. For our model, we assume that the buoyancy force acts through the center of mass of an arbitrary link $j$ and is given by the following:

$$
\mathbf{R}_{B_{j}}=-\rho V_{j} \mathbf{g}^{B}
$$

where, $\rho$ is the density of the fluid, $V_{j}$ is the volume of fluid displaced by an arbitrary link $\mathrm{j}$ and $\mathbf{g}^{B}$ is the gravity vector expressed in the $B$ th coordinate frame. The generalized active force due to buoyancy for the system is given by the following:

$$
\left(\mathbf{F}_{r}\right)_{\text {Buoy }}=-\rho \sum_{i=B}^{n} V_{i} \frac{\partial \mathbf{v}_{i}^{B}}{\partial \dot{q}_{r}} \cdot \mathbf{g}^{B}
$$

The Froude-Kriloff force is similar to the buoyancy force in that it is proportional to the fluid displaced, but is result of acceleration of the fluid itself. The force due to Froude-Kriloff also acts through the center of buoyancy and is given by:

$$
\mathbf{R}_{\mathrm{FK}_{j}}=\rho V_{i} \mathbf{a}_{f}^{B}
$$

The generalized active force due to Froude-Kriloff for the system is given by the following:

$$
\left(\mathbf{F}_{r}\right)_{\mathrm{FK}}=\rho \sum_{i=B}^{n} V_{i} \frac{\partial \mathbf{v}_{i}^{B}}{\partial \dot{q}_{r}} \cdot \mathbf{a}_{f}^{B}
$$

The fluid damping forces are divided by drag and lift force. The fluid damping forces exerted on a body depends on the square of the relative velocity of the fluid with respect to the body; the geometric shape of the body which is characterized by a drag and lift coefficient and a reference area of the body; and the density of the fluid. The drag forces include pressure drag, skin friction drag, and lift forces. The pressure drag force acts in a direction opposite to the link relative velocity with respect to the fluid and is the primary drag force for slow moving fish robot applications. Skin friction drag which is tangent to the link surface may be neglected for slow moving fish robot applications. Therefore, we will only consider drag forces could be handled for other applications in an analogous manor. The equations for the force and moment on each part due to pressure drag is given by:

$$
\begin{aligned}
& \mathbf{R}_{\operatorname{Drag}_{j}}=-0.5 \rho \int_{0}^{L} C_{D} b_{j}\left\|\mathbf{v}_{j}^{r}(l)^{\perp}\right\| \mathbf{v}_{j}^{r}(l)^{\perp} d l \\
& \mathbf{T}_{\operatorname{Drag}_{j}}=-0.5 \rho \int_{0}^{L} C_{D} b_{j}\left\|\mathbf{v}_{j}^{r}(l)^{\perp}\right\|\left(\overline{\mathbf{A}}_{B}^{j} \hat{x}_{j} \times \mathbf{v}_{j}^{r}(l)^{\perp}\right) d l
\end{aligned}
$$

where $b_{j} d l$ is the reference area of a $j$ th link, $b_{j}$ is the width of the rectangle that circumscribes the frontal projection of the infinitesimal element of a $j$ th link, and $d t$ is the length of the infinitesimal element. $C_{D}$ is the drag coefficient, and $\mathbf{v}_{j}^{r}(l)^{\perp}$ is the relative velocity of an arbitrary link $j$ with respect to the fluid normal to the part along the length, $l$, of the part. The drag coefficient $C_{D}$ is a function of part geometry and fluid flow angle. It can be represented by:

$$
C_{D}=C_{\mathrm{D}, \text { basic }} \sin ^{2} \sigma
$$

where $C_{\mathrm{D} \text {,basic }}$ is shape parameter, and $\sigma$ is the angle between the relative velocity of the fluid and the part longitudinal axis. The generalized active force due to the drag force and torque for the system is then given by:

$$
\left(\mathbf{F}_{r}\right)_{\text {Drag }}=\sum_{i=B}^{n}\left(\frac{\partial^{E} \boldsymbol{\omega}^{i}}{\partial \dot{q}_{r}} \cdot \mathbf{T}_{\operatorname{Drag}_{i}}+\frac{\partial \mathbf{v}_{i}^{B}}{\partial \dot{q}_{r}} \cdot R_{\operatorname{Drag}_{i}}\right)
$$

\subsection{Dynamic Model}

Having developed all of the generalized inertia forces and generalized active forces for the fish body, the equations of motion are found to obtain the following dynamic model:

$$
\begin{aligned}
\left(\mathbf{F}_{r}^{*}\right)+\left(\mathbf{F}_{r}^{*}\right)_{\mathrm{AM}} & +\left(\mathbf{F}_{r}\right)_{\text {gravity }}+\left(\mathbf{F}_{r}\right)_{\text {Buoy }} \\
& +\left(\mathbf{F}_{r}\right)_{\mathrm{FK}}+\left(\mathbf{F}_{r}\right)_{\text {Drag }}=0
\end{aligned}
$$

Eq.25 may not be the most convenient form for the equations of motion. Then, we make the following form:

$$
\mathbf{M}(\boldsymbol{\xi}) \ddot{q}+\mathbf{C}(\boldsymbol{\xi}, \dot{\mathbf{q}})+\mathbf{G}(\boldsymbol{\xi})+\mathbf{F}_{\text {external }}=0
$$

where $\xi$ is the joint variable vector, $\mathbf{M}(\boldsymbol{\xi})$ is the matrix of inertia term, $\mathbf{C}(\boldsymbol{\xi}, \dot{\mathbf{q}})$ is the matrix of Coriolis/Centripetal term and $\mathbf{G}(\boldsymbol{\xi})$ is the vector of gravity effect.

$$
\begin{aligned}
(\mathbf{M}(\boldsymbol{\xi})) & =-\frac{\partial\left(\left(\mathbf{F}_{i}^{*}\right)+\left(\mathbf{F}_{i}^{*}\right)_{\mathrm{AM}}\right)}{\partial \ddot{q}_{j}} \\
(\mathbf{C}(\boldsymbol{\xi}, \dot{\boldsymbol{q}})) & =-\left(\left(\mathbf{F}^{*}\right)+\left(\mathbf{F}^{*}\right)_{\mathrm{AM}}\right)-\mathbf{M}(\boldsymbol{\xi}) \ddot{\mathbf{q}} \\
\mathbf{G}(\boldsymbol{\xi}) & =-\mathbf{F}_{\text {gravity }} \\
(\mathbf{F})_{\text {external }} & =-(\mathbf{F})_{\text {Buoy }}-(\mathbf{F})_{\mathrm{FK}}-(\mathbf{F})_{\text {Damping }}
\end{aligned}
$$




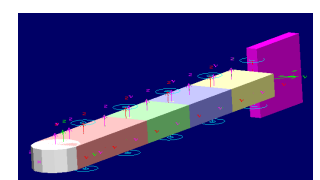

Fig. 3. Anguilliform Mode Swimming Using DADs
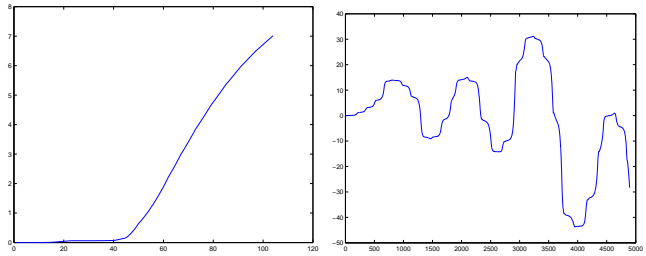

(a) $\mathrm{x}$ direction(forward) (b) y direction(turning) Fig. 4. Anguilliform Mode Swimming

\section{DYNAMIC SIMULATION FOR EACH SWIMMING MODE}

In order to compare with each swimming mode, we give the same input for each mode and examine its $\mathrm{x}$ displacement and angle about $\mathrm{x}$ axis with respect to the body(forward direction of C.M. of the body). To examine easily and optimize the hydrodynamical model for different behaviors, objective function(fitness criteria) must be selected. The long displacement about $\mathrm{x}$ direction means high forward speed(high propulsive efficiency because of the same input energy), and the large angle about $\mathrm{x}$ axis during the same time means quick turning motion.

\subsection{Anguilliform Mode}

In anguilliform mode, the whole body participates in large-amplitude undulations. Since at least one complete wavelength of the propulsive wave is present along the body, lateral forces are adequately canceled out, minimizing any tendencies for the body to recoil. Many anguilliform swimmers like eel and lamprey are capable of backward as well as forward swimming by altering the propagation direction of the propulsive wave. Backward swimming requires increased lateral displacements and body flexibility. To develop the robot, we consider that they have 6 links shown by Figure 3. Forward displacements during the same time(long displace means high forward speed) at anguilliform mode are shown Figure 4(a). Y direction displacement for quick turning motion is shown Figure 4(b). In these figures, $\mathrm{x}$ axis means the number of data, not time.

\subsection{Subcarangiform Mode}

In the subcarangiform mode(e.q., trout), the amplitude of the undulations is limited anteriorly, and increases only in the posterior half of the body. To develop the robot, we consider that

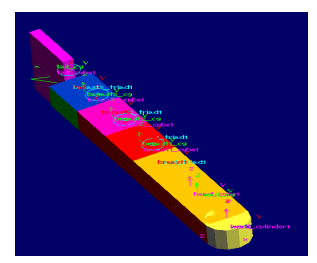

Fig. 5. Subcarangiform Mode Swimming Using DADs
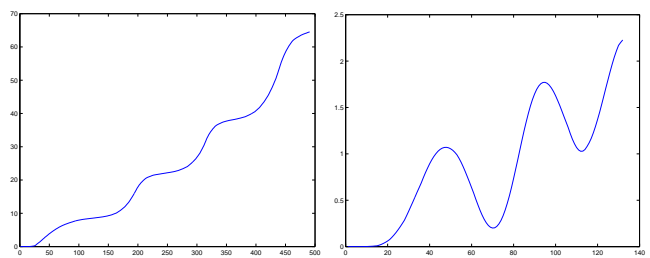

(a) $\mathrm{x}$ direction(forward) (b) y direction(turning) Fig. 6. Subcarangiform Mode Swimming

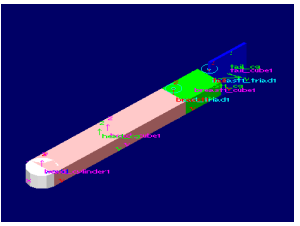

Fig. 7. Carangiform Mode Swimming Using DADs
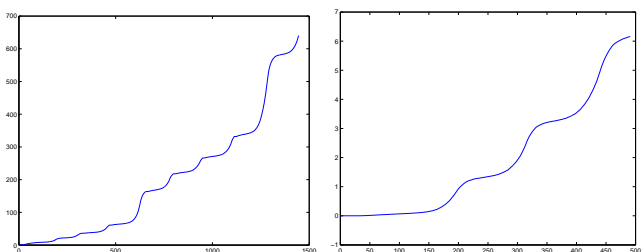

(a) $\mathrm{x}$ direction(forward) (b) y direction(turning) Fig. 8. Carangiform Mode Swimming

they have 5 links shown by Figure 5. Forward displacement is shown Figure 6(a). Y direction displacement is shown Figure 6(b).

\subsection{Carangiform Mode}

For carangiform swimming, this is even more pronounced, as the body undulations are further confined to the last third of the body length, and thrust is provided by a rather stiff caudal fin. Carangiform swimmers are generally faster than anguilliform or subcarangiform swimmers. However, their turning and accelerating abilities are compromised because of the relative rigidity of their bodies. To develop the robot, we consider that they have 3 links shown by Figure 7. Forward displacement is shown Figure 8(a). Y direction displacement is shown Figure $8(\mathrm{~b})$.

\subsection{Thunniform Mode}

Thunniform mode is the most efficient locomotion mode evolved in the aquatic environment, where thrust is generated by the lift-based method, 


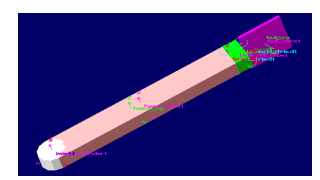

Fig. 9. Thunniform Mode Swimming Using DADs
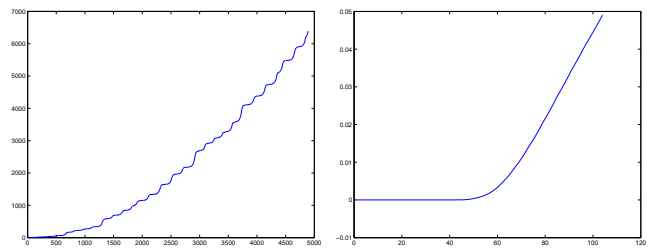

(a) $\mathrm{x}$ direction(forward) (b) y direction(turning)

Fig. 10. Thunniform Mode Swimming

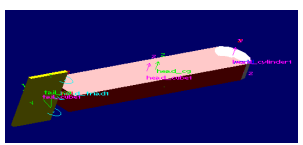

Fig. 11. Ostraciiform Mode Swimming Using DADs
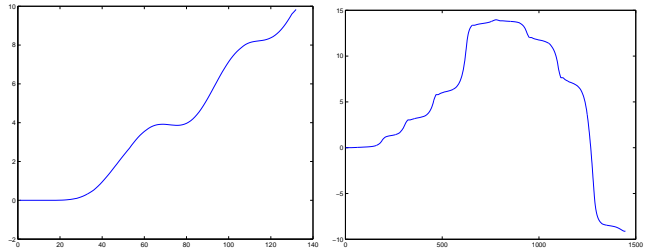

(a) $\mathrm{x}$ direction(forward) (b) y direction(turning) Fig. 12. Ostraciiform Mode Swimming

allowing high cruising speeds to be maintained for long periods. Significant lateral movements occur only at the caudal fin (that produces more than $90 \%$ of the thrust) and at the area near the narrow peduncle. To develop the robot, we consider that they have 3 links shown by Figure 9 . This is the same number of link with carangiform, but the length of peduncle is different. Forward displacement is shown Figure 10(a). Y direction displacement is shown Figure 10(b).

\subsection{Ostraciiform Mode}

Ostraciiform locomotion is the only purely oscillatory BCF mode. It is characterized by the pendulum-like oscillation of the (rather stiff) caudal fin, while the body remains essentially rigid. Fish utilizing ostraciiform mode are usually encased in inflexible bodies and forage their (usually complex) habitat using MPF propulsion. Caudal oscillations are employed as auxiliary locomotion means to aid in thrust production at higher speeds, to ensure that the body remains adequately rigid, or to air prey stalking. To develop the robot, we consider that they have 2 links shown by Figure 11. Forward displacement is shown Figure 12(a). Y direction displacement is shown Figure 12(b).

\section{CONCLUSIONS AND FUTURE WORK}

In this paper, we show the simulation result to find the suitable fish swimming modes(specially BCF swimming) for fishlike underwater robot system. We have proposed the dynamic equations for each swimming mode and the appropriate swimming mode for design by dynamic simulation. In Figure 3 - Figure 12, we can know that anguilliform swimming is appropriate mode for quick turning motion and thunniform swimming is good mode for high propulsion motion. If we have better result, we must select the optimal input signal. In this paper, we use the only harmonic signal for input. In the future, we must study to select the optimal input signal to locomotion for high speed and quick turning.

\section{REFERENCES}

[Barrett, 2002] David Barrett, "Optimization of Swimming Locomotion by Genetic Algorithm," Neurotechnology for Biomimetic Robots, pp.207-221

[Anderson, 2002] Jamie M. Anderson and Peter A. Kerrebrock, "Biomimetics in Action: Design and Performance of an Autonomous Robotic Fish," Neurotechnology for Biomimetic Robots, pp.297-308

[Budick, 2000] S.A. Budick, D.M. O'Malley, "Locomotor Repertoire of the Laval Zebrafish: Swimming, Turning and Prey Capture," Journal of Experimental Biology, 203, pp.2565-2579, 2000.

[Domenici, 1997] P. Domenici, RW. Blake, "The Kinematic and Performance of Fish FastStarting Swimming," The Journal of Experimental Biology, 200, pp.1165-1178, 1997.

[Tarn, 1996] T.J.Tarn G.A.Shoults and S.P.Yang, "A Dynamic Model of an Underwater Vehicle with a Robotic manipulator using Kane's method," Autonomous Robotics, vol. 3, 1996, pp. 267-283.

[Sfakiotakis, 1999] M.Sfakiotakis D.M.Lane and J.B.C.Davies, "Review of Fish Swimming Modes for Aquatic Locomotion," IEEE Journal of Ocean engineering, vol. 24, No. pp 237252, April 1999.

[Breder,1926] C.M. Breder, "The Locomotion of Fishes," Zoologica, vol. 4, pp 159-256, 1926.

[Kane, 1983] Thomas R. Kane, Perter W. Likins, and David A. Levinson, "Spacecraft Dynamics," McGraw-Hill Book Company, 1983.

[McMillan, 1992] Scott McMillan P.Sadayappan and David E.Orin, "Efficient Dynamic Simulation of Multiple Manipulator Systems with Singularities," ICRA, May 1992, pp. 299-304.

[Fossen, 1994] T.I.Fossen, "Guidance and Control of Ocean Vehicle," John Wiley and Sons Ltd., 1994. 